\title{
A Rare Compound Odontoma in Mandibular Midline Region: Case Report and Literature Review
}

\section{Puranik $\mathrm{CP}^{1 *}$, Mallipeddi $\mathrm{A}^{2}$ and Flaitz $\mathrm{CM}^{3}$}

${ }^{1}$ Department of Pediatric Dentistry, School of Dental Medicine and Children's Hospital Colorado, USA

${ }^{2}$ International Student Program, USA

${ }^{3}$ Department of Diagnostic \& Biological Sciences, University of Colorado, USA

\section{Case Report}

Volume 6 Issue 3

Received Date: August 30, 2021

Published Date: September 14, 2021

DOI: $10.23880 /$ oajds-16000307

*Corresponding author: Chaitanya Puranik, Director of Predoctoral Education in Pediatric Dentistry, Children's Hospital Colorado and School of Dental Medicine, University of Colorado, Anschutz Medical Campus, Aurora, Colorado-80045, USA, Fax: 720-777-7239; Tel: 720-777-2719; Email: chaitanya.puranik@childrenscolorado.org

\section{Abstract}

Background: Odontomas are the most common odontogenic tumors of the oral cavity. Although compound odontomas are common in the pediatric population, they are rare in the mandibular midline region during the primary dentition stage.

Case Description: This article provides a rare case-report and literature review of a compound odontoma in the mandibular midline region during the primary dentition stage. A routine occlusal radiograph in an asymptomatic six-year-old patient demonstrated mixed, radiolucent-radiodense lesion apical to \#N-\#P (mandibular primary incisors) with intact lamina dura. Minor expansion and thinning of labial and lingual cortices were noted on cone beam computed tomographic (CBCT) images. This report discusses surgical management, histopathologic diagnosis, and 36-month clinical-radiographic follow-up of our case.

Practical Implications: Compound odontomas are highly rare during the primary dentition stage in the mandibular midline $(0.3 \%)$. Routine radiographs are vital for a timely diagnosis and treatment of these pathologies to prevent future orthodontic complications.

Keywords: Odontoma; Compound Odontoma; Mandibular Anterior Region; Primary Dentition; Pediatric Oral Pathology

Abbreviations: CBCT: Cone-Beam Computed Tomographic

\section{Introduction}

Odontogenic tumors are rare with a reported incidence of $<0.1 \%$ [1]. An odontoma is the most common odontogenic tumor of the oral cavity comprising of $22 \%$ of all odontogenic tumors in jaws [2]. Although odontomas are usually classified as a mixed odontogenic tumor with functional ameloblasts and odontoblasts, they are best described as a developmental anomaly or hamartoma, rather than a true neoplasm [2,3]. The etiology of an odontoma is still unclear. Trauma, genetic factors, and chronic inflammation have been suggested as possible etiologic factors [1]. Complex odontoma consists of unorganized masses of dental tissue while compound odontoma consists of disordered toothlike structures $[1,2]$. Some odontomas show features of both 
types. The two types of odontomas are usually categorized based on the radiographic findings and definitively diagnosed by histopathologic examination [1]. Most of the odontomas have been diagnosed in the first two decades of life and are most common in the maxilla as compared to the mandible [1]. Odontomas affect males and females equally $[1,4]$. Odontomas are more commonly associated with permanent than primary dentition [3]. Differential diagnoses of odontoma includes cementoblastoma, osteoblastoma, calcifying odontogenic cyst, ameloblastic fibro-odontoma, osteoma and cemento-ossifying fibroma depending on the type and stage of development [4]. Treatment involves surgical removal allowing for subsequent eruption of permanent teeth and recurrence is rare [2].

Compound odontoma in primary dentition is uncommon [5] and has been commonly reported in maxillary incisors and canine regions [3]. Occurrence of compound odontoma in the mandibular incisor (midline) region is highly rare. Here, we present a unique case of a compound odontoma in the mandibular midline region during the primary dentition stage. We presented clinical and radiographic findings along with surgical management with 36-months of clinical and radiographic follow-up.

\section{Case Report}

A healthy, 6-year-old female patient presented to the Children's Hospital Colorado's Dental Center in April 2018, for a routine dental examination. Clinical examination revealed primary dentition with normal occlusion with no apparent pathoses, mobility, swelling, or pain. Generalized spacings were present between mandibular primary incisors (Figures 1A-B). Upon routine radiographic examination, a mixed (radiopaque-radiolucent) lesion surrounded by a radiolucent halo was evident on the mandibular occlusal radiograph apical to the mandibular primary incisors \#N-\#P with intact lamina dura (Figure 1C). The internal structures of the entity resembled multiple compactly arranged tooth-like formations. The developing permanent incisors were seen apical to the mixed lesion, resulting in mild root divergence of the adjacent primary incisors and inferior displacement of the developing permanent incisors. After the incidental finding, Cone-Beam Computed Tomographic (CBCT) images were taken, demonstrating minor expansion, and thinning of the labial and lingual cortical plates around the lesion (Figures 1D-G). Based on the age and presence of tooth-like formations, our differential diagnoses included compound odontoma, calcifying odontogenic cyst, and ameloblastic fibro-odontoma.

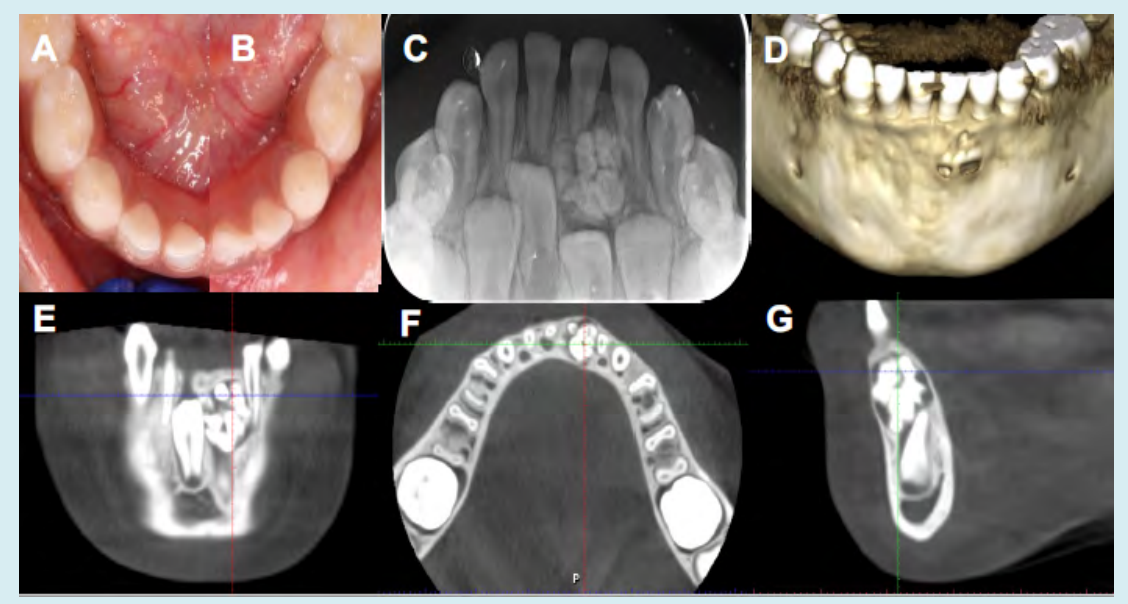

Figure 1: Clinical photograph of an occlusal view of the mandibular right quadrant (A) and left quadrant (B). Preoperative occlusal radiograph showing radiolucent-radiodense mixed lesion with demarcated margins apical to mandibular incisors (\#N-\#P) with intact lamina dura (C). Cone beam computed tomographic (CBCT) images with 3D renderings (D), coronal plane (E), axial plane $(F)$, and sagittal plane $(G)$ demonstrating the lesion with minor expansion and thinning of labial and lingual cortical plates.

A surgical treatment option was presented to the family for removal of the lesion and subsequent pathologic examination. The family provided consent for the treatment, but expressed concerns due to the patient's young age. Hence, the surgical procedure was planned under general anesthesia. During the surgical procedure, extraction of teeth \#N-\#Q was performed to allow access to the lesion. A full thickness mucoperiosteal flap was raised, and the labial cortical plate was removed using a Rongeur to expose the lesion (Figure 2A). The lesion along with the surrounding sac was removed carefully without damaging the permanent successors (Figure 2B). After hemostasis, the surgical site was closed with interrupted sutures (Figure 2C), and the patient was discharged after recovery. The patient had an uneventful healing after the surgical procedure. 
The specimen (Figure 2D) was submitted for histopathologic examination. The patient was followed for 2-, 4-, and 12-weeks after the surgical procedure with regular biannual clinical and radiographic follow-up until 36 months. At follow-up appointments, development and eruption of successor permanent incisors was evident on an occlusal radiograph without any residual pathology (Figures 2E-F). The position of lateral incisor was slightly occlusal or superior to the central incisor indicative of possible future orthodontic problems such as delayed eruption of the central incisor as seen on the panoramic radiograph (Figure 2G). Hematoxylin-Eosin staining of the decalcified mass revealed numerous atypical "tooth-like" structures, with enamel matrix, tubular dentin, pulpal components, and dental papillae with associated odontogenic epithelium (Figures 3A-B) confirming the final histological diagnosis as a compound odontoma. Parents provided consent for use of de-identified images.

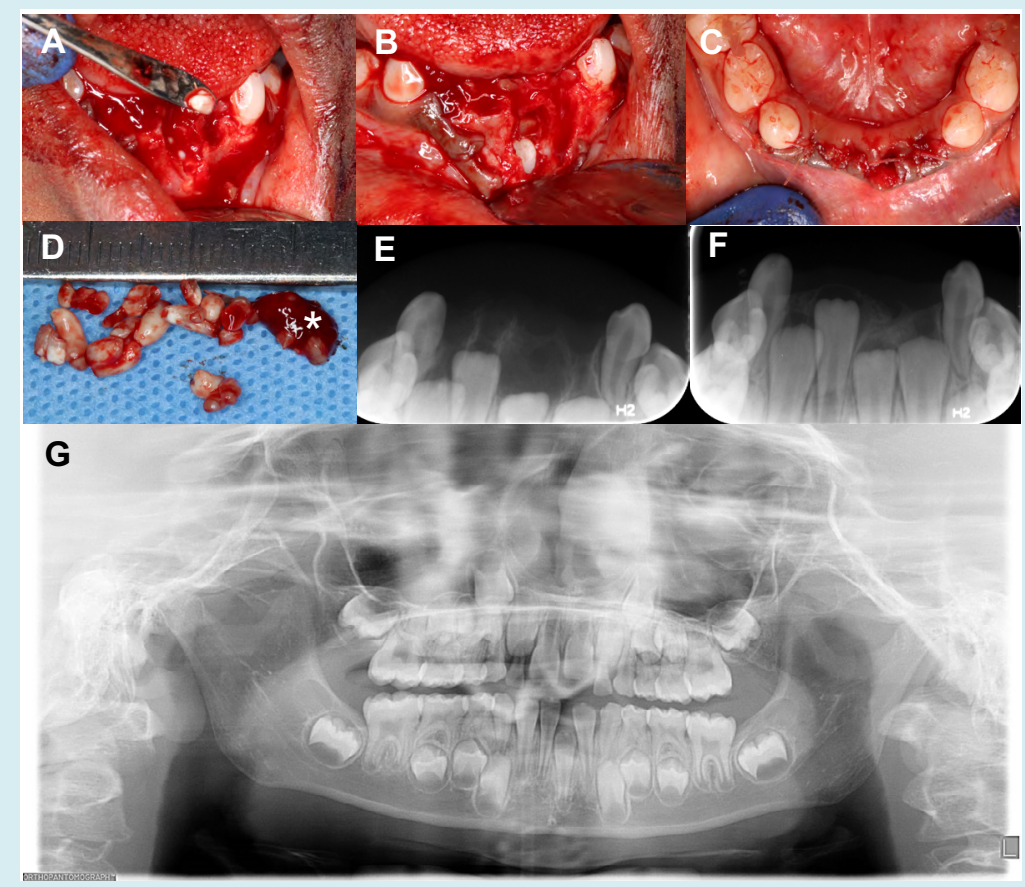

Figure 2: Intraoperative photograph after surgical site exposure and removal of calcified tissues (A), complete removal of lesion and lesion sac, coronal to the permanent tooth bud \#23 (B), surgical site closure with interrupted sutures (C), and surgically removed lesion and lesion sac $\left({ }^{*}\right)$. Postoperative occlusal radiographs at 6-months (E), and 12-months (F) demonstrating altered eruption path of permanent teeth. Panoramic radiograph (G) taken after 36-months of the surgery demonstrated delayed eruption of \#23 and \#24 as compared to the contralateral incisors.

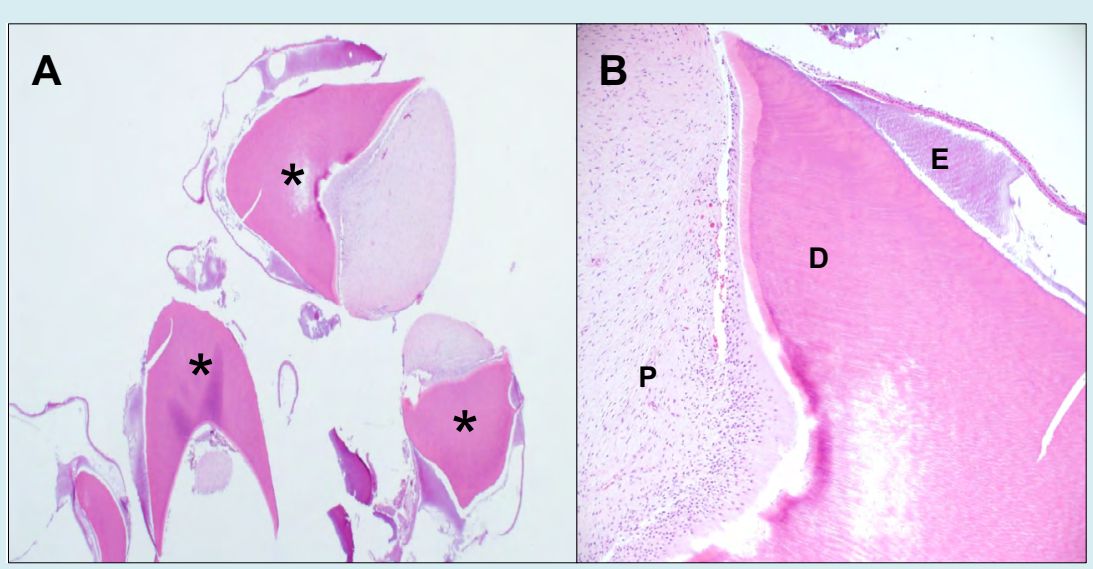

Figure 3: Hematoxylin and Eosin (H\&E) stained sections: $5 X(A)$ and 10X (B) magnification demonstrating numerous atypical "tooth-like" structures $\left({ }^{*}\right)$, enamel matrix (E), dentinal tubules (D), and dental pulp (P). 


\section{Discussion}

We performed a systematic literature review of all the published articles on Medline database searches using PubMed and Google Scholar search engines from 19752020 for reported cases of 'compound odontoma' in 'children'. Abstracts from 375 articles were reviewed to shortlist 20 articles, which are presented in table 1 [1,3-21] with 321 reported cases that met our inclusion criteria of odontomas in children and adolescents. Selected articles were systematically analyzed to identify age, sex, location, and findings associated with an odontoma. The majority of these odontoma cases were reported in the maxilla whereas, very few cases were reported in the mandible in the caninemolar region [1]. Compound odontoma constituted 53\% of reported cases only $3.1 \%$ cases were reported in the mandibular anterior region with only $0.3 \%$ of cases during the primary dentition stage. It was evident from the literature review (Table 1) that compound odontoma is rare in the mandibular midline region and highly rare during primary or mixed dentition stages. These findings from the literature review emphasize that our case was exceptionally rare.

\begin{tabular}{|c|c|c|c|c|c|c|c|}
\hline \multicolumn{8}{|c|}{ Table 1: Reported Cases of Compound Odontoma in Children } \\
\hline Author & Year & Study Design & $\begin{array}{l}\text { Sample } \\
\text { size }\end{array}$ & $\begin{array}{c}\text { Age } \\
\text { (years) }\end{array}$ & $\begin{array}{c}\text { Sex } \\
\text { (M: male } \\
\text { F: female) }\end{array}$ & Type & Common Location \\
\hline Budnick [6] & 1976 & $\begin{array}{l}\text { Literature } \\
\text { review }\end{array}$ & 149 & $10-19$ & M & $\begin{array}{l}\text { Complex } \\
\text { and } \\
\text { compound }\end{array}$ & $\begin{array}{c}\text { Complex: Posterior } \\
\text { (Maxilla, Mandible); } \\
\text { Compound: } \\
\text { Maxillary Anterior }\end{array}$ \\
\hline \multirow{3}{*}{ Brunetto, et al. [7] } & \multirow{3}{*}{1991} & \multirow{3}{*}{ Case series } & \multirow{3}{*}{3} & 4 & $\mathrm{M}$ & Compound & Maxillary Canine \\
\hline & & & & 12 & $\mathrm{~F}$ & Compound & $\begin{array}{l}\text { Maxillary Incisors } \\
\text { and Canine }\end{array}$ \\
\hline & & & & 11 & $\mathrm{M}$ & Compound & Maxillary Incisor \\
\hline de Oliveira, et al. [8] & 2001 & Case report & 3 & 5 & $\mathrm{~F}$ & Compound & Maxillary Incisor \\
\hline Das, et al. [9] & 2002 & Case report & 1 & 10 & $\mathrm{~F}$ & Compound & Maxillary Canine \\
\hline Yeung, et al. [10] & 2003 & Case report & 1 & 2 & M & Compound & Maxillary Incisor \\
\hline \multirow{17}{*}{ Sheehy, et al. [4] } & \multirow{17}{*}{2004} & \multirow{17}{*}{$\begin{array}{l}\text { Literature } \\
\text { review \& Case } \\
\text { series }\end{array}$} & \multirow{17}{*}{16} & 4 & $\mathrm{~F}$ & Complex & Maxillary Canine \\
\hline & & & & 4 & $\mathrm{M}$ & Compound & Maxillary Canine \\
\hline & & & & 4 & M & Compound & $\begin{array}{l}\text { Maxillary Incisor, } \\
\text { Canine, Molar }\end{array}$ \\
\hline & & & & 3 & M & Compound & $\begin{array}{c}\text { Maxillary Incisor, } \\
\text { Canine, Molar }\end{array}$ \\
\hline & & & & 4 & M & Complex & Mandibular Incisor \\
\hline & & & & 4 & $\mathrm{M}$ & Compound & Maxillary Canine \\
\hline & & & & 8 & $\mathrm{~F}$ & Compound & Maxillary Canine \\
\hline & & & & 5 & $\mathrm{~F}$ & Compound & Maxillary Canine \\
\hline & & & & 6 & $\mathrm{M}$ & Compound & Maxillary Incisor \\
\hline & & & & 2 & $\mathrm{~F}$ & Compound & Maxillary Canine \\
\hline & & & & 1 & $\mathrm{~F}$ & Compound & Maxillary Incisor \\
\hline & & & & 1 & $\mathrm{M}$ & Compound & Maxillary Incisor \\
\hline & & & & 3 & $\mathrm{~F}$ & Compound & Maxillary Canine \\
\hline & & & & 3 & $\mathrm{~F}$ & Compound & Maxillary Incisor \\
\hline & & & & 2 & $\mathrm{M}$ & Compound & Maxillary Molar \\
\hline & & & & 3 & $\mathrm{~F}$ & Complex & Maxillary Molar \\
\hline & & & & 4 & $\mathrm{M}$ & Compound & Maxillary Canine \\
\hline
\end{tabular}


Open Access Journal of Dental Sciences

\begin{tabular}{|c|c|c|c|c|c|c|c|}
\hline \multirow{2}{*}{ Delbem, et al. [11] } & \multirow{2}{*}{2005} & \multirow{2}{*}{ Case series } & \multirow{2}{*}{2} & 5 & $\mathrm{M}$ & Compound & Maxillary Canine \\
\hline & & & & 2 & $\mathrm{M}$ & Compound & Maxillary Incisor \\
\hline Clidir, et al. [2] & 2005 & Case report & 1 & 8 & $\mathrm{~F}$ & Compound & Mandibular Canine \\
\hline \multirow[b]{2}{*}{ Yildirim-Oz, et al. [3] } & \multirow[b]{2}{*}{2007} & \multirow[b]{2}{*}{ Case series } & \multirow[b]{2}{*}{2} & 4 & $\mathrm{~F}$ & Compound & Mandibular Canine \\
\hline & & & & 13 & $\mathrm{~F}$ & Compound & Mandibular Canine \\
\hline Aizenbud, et al. [12] & 2008 & Case report & 1 & 5.5 & $\mathrm{M}$ & Compound & Maxillary Incisors \\
\hline Teruhisa, et al. [13] & 2009 & Case report & 1 & 6 & $\mathrm{~F}$ & Compound & Mandibular Molar \\
\hline \multirow{26}{*}{ Iatrou, et al. [14] } & \multirow{26}{*}{2010} & \multirow{26}{*}{$\begin{array}{l}\text { Retrospective } \\
\text { study }\end{array}$} & \multirow{26}{*}{26} & 13 & $\mathrm{~F}$ & Complex & Maxillary Incisor \\
\hline & & & & 9 & $\mathrm{~F}$ & Compound & Maxillary Incisor \\
\hline & & & & 14 & $\mathrm{M}$ & Compound & Mandibular Incisor \\
\hline & & & & 14 & $\mathrm{M}$ & Complex & Maxillary Molar \\
\hline & & & & 12 & $\mathrm{M}$ & Complex & Mandibular Molar \\
\hline & & & & 4 & $\mathrm{M}$ & Complex & Maxillary Incisor \\
\hline & & & & 8 & $\mathrm{~F}$ & Complex & Mandibular Molar \\
\hline & & & & 9 & $\mathrm{~F}$ & Compound & Maxillary Incisor \\
\hline & & & & 4 & $\mathrm{~F}$ & Compound & Mandibular Molar \\
\hline & & & & 13 & M & Compound & Maxillary Incisor \\
\hline & & & & 8 & $\mathrm{~F}$ & Compound & Maxillary Incisor \\
\hline & & & & 13 & $\mathrm{~F}$ & Complex & Mandibular Canine \\
\hline & & & & 2.5 & $\mathrm{~F}$ & Compound & Maxillary canine \\
\hline & & & & 4 & $\mathrm{M}$ & Compound & Maxillary Molar \\
\hline & & & & 11 & $\mathrm{~F}$ & Complex & Maxillary Incisor \\
\hline & & & & 11 & $\mathrm{~F}$ & Compound & $\begin{array}{l}\text { Mandibular } \\
\text { Premolar }\end{array}$ \\
\hline & & & & 8 & $\mathrm{M}$ & Compound & Maxillary Incisor \\
\hline & & & & 9 & $\mathrm{M}$ & Compound & Mandibular Molar \\
\hline & & & & 2 & M & Complex & Mandibular Molar \\
\hline & & & & 8 & $\mathrm{M}$ & Compound & Maxillary Incisor \\
\hline & & & & 8 & M & Compound & Mandibular Molar \\
\hline & & & & 11 & $\mathrm{~F}$ & Complex & Mandibular Canine \\
\hline & & & & 12 & $\mathrm{~F}$ & Complex & Maxillary Canine \\
\hline & & & & 8 & $\mathrm{M}$ & Compound & Maxillary Incisor \\
\hline & & & & 13 & $\mathrm{M}$ & Compound & Mandibular Molar \\
\hline & & & & 14 & M & Complex & $\begin{array}{c}\text { Mandibular Incisor } \\
\text { and Canine }\end{array}$ \\
\hline \multirow{5}{*}{ Conti, et al. [15] } & \multirow{5}{*}{2012} & \multirow{5}{*}{ Case series } & \multirow{5}{*}{5} & 13 & $\mathrm{~F}$ & Compound & Maxillary Canine \\
\hline & & & & 16 & $\mathrm{~F}$ & Compound & Maxillary Canine \\
\hline & & & & 5 & $\mathrm{M}$ & Compound & Maxillary Canine \\
\hline & & & & 3 & $\mathrm{~F}$ & Compound & Maxillary Canine \\
\hline & & & & 6 & $\mathrm{~F}$ & Compound & Maxillary Incisors \\
\hline Mathew, et al. [16] & 2013 & Case report & 1 & 4 & $\mathrm{~F}$ & Compound & Maxillary Incisors \\
\hline Poleti, et al. [17] & 2013 & Case report & 1 & 3 & $\mathrm{~F}$ & Compound & Maxillary Canine \\
\hline Hanemann, et al. [18] & 2013 & Case report & 1 & 15 & $\mathrm{~F}$ & Compound & $\begin{array}{l}\text { Maxillary Incisor } \\
\text { and Canine }\end{array}$ \\
\hline Altay, et al. [5] & 2016 & Case report & 1 & 5.5 & $\mathrm{~F}$ & Compound & Maxillary Incisors \\
\hline
\end{tabular}




\begin{tabular}{|c|c|c|c|c|c|c|c|}
\hline Soluk, et al. [19] & 2016 & $\begin{array}{c}\text { Literature } \\
\text { review }\end{array}$ & 60 & $0-17$ & $\mathrm{M}$ & $\begin{array}{c}\text { Complex: } \\
\text { Mandibular } \\
\text { Posterior; } \\
\text { Complex } \\
\text { and } \\
\text { compound }\end{array}$ \\
\hline Isola, et al. [1] & 2017 & $\begin{array}{c}\text { Retround: } \\
\text { Maxillary Anterior } \\
\text { and } 6 \text { cases in } \\
\text { mandibular } \\
\text { anterior region }\end{array}$ \\
\hline Uma, et al. [20] & 2017 & Case report & 1 & 9 & $\mathrm{~F}$ & Compound & Mandibular Incisor \\
\hline
\end{tabular}

Table 1: Literature review of all the reported cases of compound odontoma in children during primary, mixed, and permanent dentition stages.

Although most compound odontomas are asymptomatic, the common signs and symptoms associated with compound odontoma are eruption disturbances, pain, and swelling $[1,4]$. Odontomas usually cause disturbances in the position of teeth (including tipping or displacement of the adjacent teeth) and changes in the eruption patterns (including delayed eruption, impacted teeth, and retained primary teeth) [2]. In our case too, central and lateral incisors (\#24 and \#23, respectively) seemed to be moved away from their normal path of eruption causing delayed eruption as compared to the contralateral teeth (\#25 and \#26). More than half of the reported cases of compound odontoma were associated with delayed eruption of permanent teeth, while approximately one-fourth of the cases demonstrated no signs or symptoms [1] or cortical expansion [5]. In our case, no signs or symptoms were reported except for the minor cortical expansion and thinning of the labial and lingual cortical plates as detected on the CBCT reconstructed images. Complication after surgical removal of the odontoma include delayed eruption and impaction of permanent teeth, which may need orthodontic treatment in the future. Surgical trauma to the developing permanent tooth bud could lead to changes in the eruption path or dilaceration of the permanent tooth root [5]. Due to the early intervention in our case, we have not seen dilacerations of the permanent mandibular incisors.

Compound odontoma is usually an incidental finding on routine radiographic examination [1-5]. Hence, routine radiographs during primary dentition are crucial for evaluation of potential odontogenic findings such as the odontoma $[1,5]$. Common radiographic findings in compound odontomas include multiple, well-defined radiopacities in the alveolar bone surrounded by a radiolucent halo [3]. Radiographically, lesions may have thin, sclerotic margins with variably-sized tooth-like radiopacities [2]. Depending on the developmental status, the lesion may appear as radiolucent, mixed, radiopaque-radiolucent (partially calcified) or radiopaque with a surrounding radiolucent halo. As seen in our case, compound odontomas associated with primary teeth are less radiopaque as compared to those associated with permanent teeth [3]. If untreated, cystic transformation of an odontoma has been reported [4]. Odontomas may also be associated with lesions like dentigerous cyst, calcifying odontogenic cyst and ameloblastic fibro-odontoma [1,4].

\section{Clinical Implications}

Development and maturation of odontomas usually occur concurrently with the development of adjacent teeth [4]. It is uncommon to detect odontogenic pathosis in primary dentition [1]. However, early detection could allow for conservative management through minimally traumatic surgical intervention with fewer complications during permanent dentition stage. Routinely, bitewings and periapical radiographs are taken in primary and mixed dentition stages for diagnosis of interproximal dental caries and periapical disease. This case report emphasizes the value of taking occlusal radiographs in primary dentition along with bitewings and periapical radiographs to rule out any pathology or incidental findings such as odontomas in children.

\section{Acknowledgement}

We would like to acknowledge the contribution of Dr. Mark Berman for his help with the surgical consult and procedure.

\section{References}

1. Isola G, Cicciù M, Fiorillo L, Matarese G (2017) Association between Odontoma and Impacted Teeth. J Craniofac Surg 28(3): 755-758.

2. Cildir SK, Sencift K, Olgac V, Sandalli N (2005) Delayed eruption of a mandibular primary cuspid associated with compound odontoma. J Contemp Dent Pract 6(4): 


\section{Open Access Journal of Dental Sciences}

152-159.

3. Yildirim Oz G, Tosun G, Kiziloglu D, Durmuş E, Sener $Y$ (2007) An unusual association of odontomas with primary teeth. Eur J Dent 1(1): 45-49.

4. Sheehy EC, Odell EW, Al Jaddir G (2004) Odontomas in the primary dentition: literature review and case report. J Dent Child (Chic) 71(1): 73-76.

5. Altay MA, Ozgur B, Cehreli ZC (2016) Management of a Compound Odontoma in the Primary Dentition. J Dent Child (Chic) 83(2): 98-101.

6. Budnick SD (1976) Compound and complex odontomas. Oral Surg Oral Med Oral Pathol 42(4): 501-506.

7. Brunetto AR, Turley PK, Brunetto AP, Regattieri LR, Nicolau GV (1991) Impaction of a primary maxillary canine by an odontoma: surgical and orthodontic management. Pediatr Dent 13(5): 301-302.

8. de Oliveira BH, Campos V, Marçal S (2001) Compound odontoma--diagnosis and treatment: three case reports. Pediatr Dent 23(2): 151-157.

9. Das UM, Arathi N (2002) Unerupted maxillary primary canine associated with compound composite odontoma: a case report. J Indian Soc Pedod Prev Dent 20(3): 98101.

10. Yeung KH, Cheung RCT, Tsang MMH (2003) Compound odontoma associated with an unerupted and dilacerated maxillary primary central incisor in a young patient. Int J Paediatr Dent 13(3): 208-212.

11. Delbem ACB, Cunha RF, Bianco KG, Afonso RL, Gonçalves TC (2005) Odontomas in pediatric dentistry: report of two cases. J Clin Pediatr Dent 30(2): 157-160.

12. Aizenbud D, Front YP (2008) An impacted malformed primary maxillary central incisor diagnosed as a compound odontoma. J Clin Pediatr Dent 33(2): 161165.

13. Teruhisa U, Murakami J, Hisatomi M, Yanagi Y, Asaumi JI (2009) A case of unerupted lower primary second molar associated with compound odontoma. Open Dent J 3: 173-176.

14. Iatrou I, Vardas E, Theologie Lygidakis N, Leventis M (2010) A retrospective analysis of the characteristics, treatment and follow-up of 26 odontomas in Greek children. J Oral Sci 52(3): 439-447.

15. Conti G, Franchi L, Camporesi M, Defraia E (2012) Treatment protocol for the impaction of deciduous maxillary anterior teeth due to compound odontoma. Eur J Paediatr Dent 13(4): 337-341.

16. Mathew A, Shenai P, Chatra L, Veena K, Rao P, et al. (2013) Compound odontoma in deciduous dentition. Ann Med Health Sci Res 3(2): 285-287.

17. Poleti ML, Duarte BG, Lara VS, Freitas Faria P, Rubira Bullen IRF, et al. (2013) Odontoma associated with calcifying cystic odontogenic tumor in deciduous dentition: case report. Oral Maxillofac Surg 17(1): 77-80.

18. Hanemann JAC, Oliveira DT, Garcia NG, Santos MRG, Pereira AAC (2013) Peripheral compound odontoma erupting in the gingiva. Head Face Med 9: 15.

19. Soluk Tekkesin M, Tuna EB, Olgac V, Aksakallı N, Alatlı $C$ (2016) Odontogenic lesions in a pediatric population: Review of the literature and presentation of 745 cases. Int J Pediatr Otorhinolaryngol 86: 196-199.

20. Uma E (2017) Compound Odontoma in Anterior Mandible-A Case Report. Malays J Med Sci 24(3): 92-95.

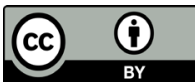

